\title{
Higher-Level Information Aspects of Web Systems: Addressing the Problem of Disconnection*
}

\author{
Farooque Azam**, Zhang Li, and Rashid Ahmad \\ Software Engineering Institute, Beijing University of Aeronautics and Astronautics \\ No.37, XueYuan Road, HaiDian District, Beijing 100083, P.R. China \\ \{farooque, lily,r.ahmad\}@buaa.edu.cn
}

\begin{abstract}
Current Web modelling languages fail to adequately support higherlevel information aspects of Web systems. Researchers have argued that there is a disconnection between functional architecture and information architecture, and also disconnection between business models and technical architectures. Addressing the problem; Web Information Exchange Diagram is developed in two flavours, WIED and WIED-UML. WIED-UML is developed to primarily address the problem of disconnection between functional architecture and information architecture. However, we argue that it, doesn't really address the issue, because WIED-UML is developed on a set of different UML - Unified Modelling Language notations which don't have semantic conformity with current UML metamodel. Creating transformation rules between models not conforming to common metamodel vocabulary, would not lead to an elegant solution. Hence in this paper, we propose a solution, by identifying linkages with two standard UML compliant approaches namely Enterprise Distributed Object Computing (EDOC) Profile and standard UML2.0 notations.
\end{abstract}

\section{Introduction}

The ability to reliably and consistently develop systems that utilise Internet and Web technologies has become increasingly important. These systems are typically, both functionally complex and information-rich [1]. In recent years, various approaches have been developed or adapted for representing these complex Web systems with different objectives. For example, the $\mathrm{e}^{3}$-value ${ }^{\mathrm{TM}}$ business modelling method [2] emphasizes a business modelling perspective, UML [3] focuses on functional aspects, and WebML [4] concentrates on the informational aspects of Web systems.

One aspect that has received increasing attention is information modelling for these applications, particularly with respect to aspects such as navigation models and their relationships to the underlying content. Information modelling approaches, such as RMM [5] and OOHDM [6], and various adaptations of UML, have provided the ability to model the contents of these applications, and the way in which we interact with this information. A good discussion on these modelling approaches is given in [1], where it has been argued that, these models have typically focussed on modelling at a relatively low-level, and have failed to address higher-level aspects, such as architectural and even business process modelling.

\footnotetext{
"Supported by Beijing Municipal Science \& Technology New Star Plan (H013610270112)

** Corresponding author: Tel: +86-10-82753679
} 
Several different levels of modelling that might typically occur in representing the design of Web-enabled systems is illustrated in Fig. 1 (from [1]).

- At the top level we can model the actual business (business goals, business processes, etc.) by utilising these systems. A typical example is the $\mathrm{e}^{3}-$ value $^{\mathrm{TM}}$ business modelling notation [2].

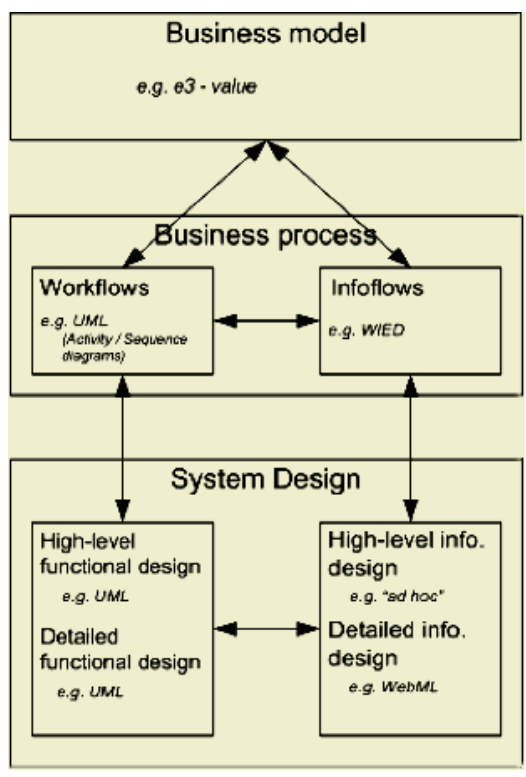

Fig. 1. Evaluation of modelling approaches [1]

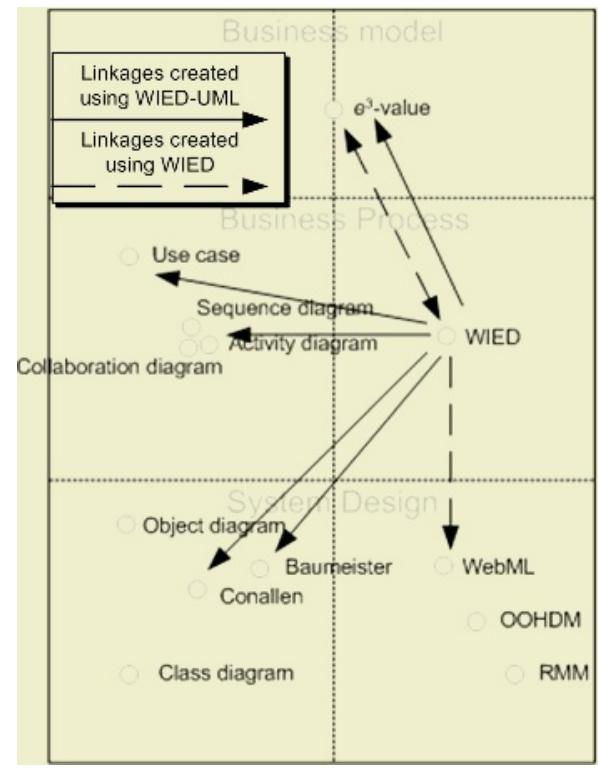

Fig. 2. Linkages between WIED-UML and other UML Models [7]

- At the middle level in this diagram are models of the high-level system architecture that capture the domains of functionality and the domains of information that are needed to support the business domains. Functional aspects at this level are well supported by UML as some UML models (such as activity diagrams) can be used to represent the business and operational workflows of a system. However, UML support for informational aspects is relatively immature; and to serve this aspect, recently WIED-Web Information Exchange Diagram [1] is developed.

- At the lowest level in this diagram are models of the detailed design. These models typically capture design elements that have a direct correspondence to specific implementation artefacts. For example, functional elements (such as code modules and communication interfaces) and information elements (such as page content and navigational links).

WIED is interesting because, researchers have indicated [8] that there is a disconnection between functional architecture and information architecture, and also disconnection between business models and technical architectures, which is the main focus of the WIED concept. However, the WIED concept is still generic and needs more effort to make it a practical companion to existing widely-used UML models and furthermore MDA-Model Driven Architecture [9] compliant approaches. 
In this paper, Section2 presents the WIED concept, its allied problems and proposed solutions. Next, moving towards the solution; we briefly present two modelling notations. These notations include, firstly, Object Management Group (OMG)'s Enterprise Distributed Object Computing (EDOC) Profile [10] in Section 3, and secondly, UML 2.0 [11] notations in Section 4. Lastly, Section 5 concludes our work.

\section{WIED: Need for Refinement}

WIED primarily forms a bridge between two existing modelling languages $e^{3}$-value $e^{\mathrm{TM}}$ (representing business value exchanges) and WebML (representing low-level information designs). These are depicted by dashed arrows showing linkages in Fig. 2 (from [7]). Resultantly, it intends to resolve the problem of disconnection between business models and information architectures. However, WIED-UML [12] has been developed and linked to various UML models and adaptations for addressing the problem of disconnection between functional architecture and information architecture, because UML comprises well-established notations for representing functional aspects. These linkages are depicted by solid arrows in Fig. 2 (see [7] for details). Nevertheless, WIED-UML is associated with a problem:

- The Problem: Resolving the problem of disconnection between functional architecture and information architecture, using WIED-UML doesn't really address the issue, because WIED-UML is developed on a set of different UML like notations as also recognised by the authors [12]. These notations don't have semantic conformity with UML metamodel. Moreover, when moving towards developing automated tools, we need transformations rules between WIED-UML and other models according to identified linkages, which however, will not provide elegant solution because the relevant models don't share the common metamodel vocabulary.

- Proposed Solution: The solution to the problem of disconnection, as we perceive, is (1) Finding all possible direct linkages without using WIED-UML; between $g e$ neric WIED model and notations representing functional architecture. One possible linkage using EDOC Profile is briefly presented in Section 3. (2) Representing WIED concept in standard UML compliant notations and further linking these notations to various other UML models, adaptations and MDA compliant approaches. In Section 4, we briefly present a possibility of using standard UML 2.0 notations for describing the WIED concept. We argue that, these linkages would allow us to move forward towards integration and interoperability of WIED concept to MDA compliant tools for achieving future goal of full lifecycle Web application development.

\section{Linking EDOC Profile and WIED}

The vision of the EDOC Profile is to simplify the development of component based EDOC systems by means of a modelling framework, based on UML 1.4, conforming MDA specifications (for details see Part I, Section I-Vision in [10]). EDOC profile comprises various specifications: Among them is the Enterprise Collaboration Archi- 
tecture (ECA) that is a technology independent profile allowing the definition of PIMs - Platform Independent Models. ECA further comprises a set of five UML profiles: (1) Component Collaboration Architecture (CCA) (2) The Entities profile (3) The Events profile (4) The Business Process Profile (BPP) (5) The Relationships profile. Among these profiles, in the context of WIED, BPP is relatively more suitable for mapping because, it specialize CCA, and describes a set of UML extensions those may be used on their own, or in combination with the other EDOC elements, to model system behaviour in the context of the business. Moreover, BPP being specialization of CCA, has inherent capability of modelling the behaviour of the components at varying and mixed levels of granularity.

EDOC Profile is interesting because it is an adopted specification of OMG and most importantly, it comprises the notations for describing PIMs as well as Platform Specific Models (PSMs) encompassing all the three modelling levels as described in Fig. 1. Although being profile of UML it tends to be more expressive of functional aspects. Conversely, WIED is also analogous to PIM because it describes informational aspects at the business process level without revealing platform specific details.

Linking WIED to BPP notations entails two significant benefits. Firstly, we get the ability to form a valuable horizontal linkage at business process level (Fig. 2) between informational and functional flows without using WIED-UML. Secondly, once a model is transformed from WIED to BPP notations, subsequently vertical mapping between the models (PIMs and PSMs) can be achieved at all the three levels by means of transformations using various EDOC profiles. Furthermore, EDOC profiles have been developed on the notion of modelling applications according to MDA specification. Hence, we contend that linking WIED to BPP notations can be considered as integration of WIED with MDA compliant approach, which will help to further augment the WIED concept.

This subset of BPP notations used for describing linkage with WIED is depicted in Fig. 3. While creating the linkage, we have defined these mappings in two phases; In Phase-I, we create an Organisation Collaboration Model that gives a broader picture of the business process between organisations. In Phase-II, we create System Collaboration Model by zooming-in the sub-activities, and subsequently depict internal information flows of the System under consideration. We have carried out a complete analysis of this mapping process and found that it can be depicted elegantly using $\mathrm{BPP}$ in a compact form.

\section{Using UML 2.0 for Representing the WIED Concept}

UML 2.0 Superstructure specifications [11] also provide promising possibility to model informational aspects at higher-level of abstraction. In the context of WIED, the most interesting facility that can be used is the package InformationFlows (see Section 17.2 pp. 665 of [11]). The document clearly describes that the InformationFlows package provides mechanisms for specifying the exchange of information between entities of a system at a high level of abstraction. They do not specify the nature of the information, nor the mechanisms by which this information is conveyed. Hence InformationFlows package has natural linkage to the WIED concept which models information flows at higher-level of abstraction. 
An information flow is represented as a dependency, with the keyword $<<$ flow $>>$ (Fig. 4) conveying InformationItem (e.g. product and wage) from source to target (e.g. Company to Customer). Semantically, sources and targets of the information flow can be kind-of Actor, Node, UseCase, Artifact, Class, Component, Port, Property, Interface, Package, and InstanceSpecification.

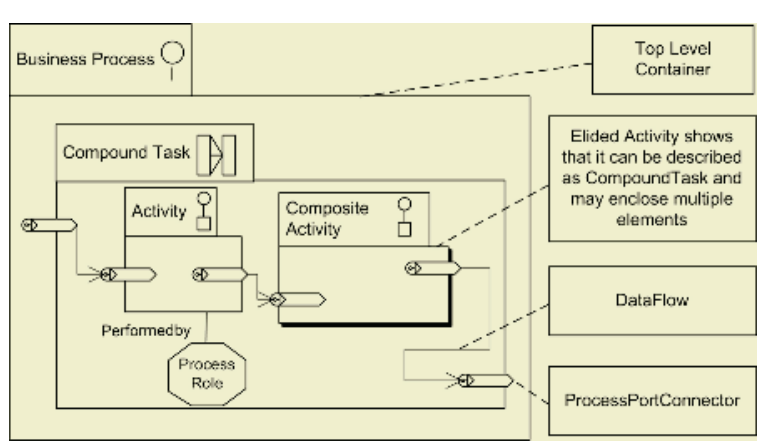

Fig. 3. Subset of EDOC's Business Process Profile notations

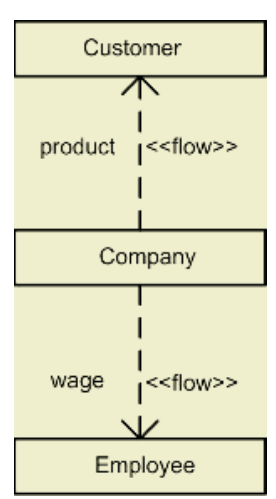

Fig. 4. Information flows conveying information items [11]

For representing the WIED concept in UML 2.0 notations, we can map various kinds of information units in WIED to appropriately stereotyped Class diagrams on similar rules defined for WIED-UML in [12]. This would also include Derivation Units those are mapped in WIED-UML differently. However, WIED-UML depicts, information flows by an arrow (lacking any UML semantics) that has resultantly created new UML diagrams. Instead, by using InformationFlows introduced in UML 2.0, we can elegantly map information flows represented in WIED using dependency stereotyped by a keyword $<<$ flow $>>$ as depicted in Fig. 4.

\section{Conclusion and Future Work}

In this paper, firstly we introduce the WIED and WIED-UML context and highlight the problem associated with WIED-UML. Next we propose the solution. Subsequently, inline with the proposed solution, we identify two methods for representing higher-level information flows, i.e., using (1) EDOC's Business Process Profile and (2) InformationFlows introduced in UML 2.0. We argue that linking these notations' augments the WIED concept and brings it closer to the MDA vision, allowing a step forward towards integration and interoperability with future MDA compliant tools.

In this paper, we have very briefly presented the proposed solutions. However, we recognize that these linkages should be demonstrated comprehensively so that researchers/practitioners can comfortably adopt these. However, paper size limitation precludes us to do so and therefore we intend to publish it elsewhere.

This paper is the outcome of ongoing work for refining the WIED concept, so that it can further be integrated with ongoing global effort of standardising modelling and model transformation approaches. 


\section{References}

1. Tongrungrojana, Rachatrin and Lowe, David.: WIED: A Web Modelling Language for Modelling Architectural-Level Information Flows. Journal of Digital Information, Volume 5 Issue 2, Article No. 283, 2004-08-10 http://jodi.ecs.soton.ac.uk/Articles/v05/i02/ Tongrungrojana/

2. Gordijn, J.: $\mathrm{e}^{3}$-value in a Nutshell. In Proceedings of International Workshop on E-business Modeling, Lausanne http://www.cs.vu.nl/ gordijn/bmns.pdf

3. Booch, G., Rumbaugh, J. and Jacobson, I.: The Unified Modelling Language User Guide (Addison-Wesley)

4. Ceri, S., Fraternali, P. and Bongio, A.: Web Modeling Language (WebML): a modeling language for designing Web sites. In Proceedings of the ninth International World Wide Web Conference, Amsterdam, May, pp. 137-157 http://www9.org/w9cdrom/177/177.html

5. Isakowitz, T., Stohr, E. and Balasubramanian, P.: RMM: A Methodology for Structured Hypermedia Design. Communications of the ACM, Vol. 38, No. 8, 34-44

6. Schwabe, D. and Rossi, G.: Developing Hypermedia Applications using OOHDM. In Proceedings of Workshop on Hypermedia Development Processes, Methods and Models (Hypertext'98), Pittsburgh

7. Tongrungrojana, R. and Lowe, D.: Forming Linkages from WIED-UML to UML Modeling System. In AusWeb04: The Tenth Australian World Wide Web Conference (Eds, Treloar, A. and Ellis, A.) Southern Cross University, Gold Coast, Australia, 3-7 July 2004, pp. 288300.

8. Gu, A., B. Henderson-Sellers, et al: Web Modelling Languages: The Gap between Requirements and Current. Exemplars. AusWeb02, Gold Coast, Australia.

9. OMG, Model Driven Architecture, A Technical Perspective, Document ab/21001-02-05, Februray 2001, http://www.omg.org

10. OMG, UML Profile for Enterprise Distributed Object Computing, Document ptc/2001-1204, December 2001

11. UML 2.0 Superstructure Final Revised Adopted specification http://www.omg.org/cgi-bin/doc?ptc/2004-10-02

12. Lowe, D. and R. Tongrungrojana: Web Information Exchange Diagrams for UML. The Fourth International Conference on Web Engineering, Munich, Germany. 